\title{
O CONSUMO COLABORATIVO NO CONTEXTO SOCIOECONÔMICO DE VULNERABILIDADE SOCIAL: 0 CASO DA COMUNIDADE PEQUENOS PROFETAS
}

\section{Denys Marques de Oliveira}

Universidade Federal Rural de Pernambuco/ UFRPE

Recife - Pernambuco - Brasil

E-mail:webdenys@gmail.com

ORCID: https://orcid.org/0000-0003-0392-2820

\section{Angela Cristina Rocha de Souza}

Universidade Federal Rural de Pernambuco/UFRPE

Recife - Pernambuco - Brasil

E-mail: angela.souza@ufrpe.br

ORCID: https://orcid.org/0000-0001-6349-5168

Recebido em 01/12/2020 aprovado em 06/05/2021.

DOI: http://dx.doi.org/10.5380/guaju.v7i1.78147

\section{Resumo}

O presente artigo tem como objetivo investigar como o consumo colaborativo modifica o contexto social de pessoas em situação socioeconômica de vulnerabilidade social. Realizamos uma pesquisa descritiva, abordando o problema de forma qualitativa e utilizando como objeto de estudo o caso da Comunidade Pequenos Profetas (CPP). Os dados foram coletados por meio de entrevistas semiestruturadas, no site da Comunidade e no relatório de atividades da Comunidade de 2018. A análise dos dados foi realizada por meio da técnica de análise de conteúdo. Os resultados demonstraram que as práticas de consumo colaborativo, tais como: reuso, produção compartilhada de bens de consumo, consumo com foco na sustentabilidade, redução de desperdício, desenvolvidas pelos participantes do caso estudado têm contribuído para modificar o contexto socioeconômico dos participantes. Isso foi observado na melhoria da qualidade de vida e nutricional dos participantes, processo de geração de renda nas famílias, fomento ao empreendedorismo e pertencimento social. Assim, concluímos que o consumo colaborativo pode modificar de forma positiva o contexto socioeconômico de pessoas em situação de vulnerabilidade social.

Palavras-chave: Economia colaborativa. Mudança social. Consumo sustentável. 


\title{
Collaborative Consumption in the Socioeconomic Context of Social Vulnerability: the Case of the Community of Little Prophets
}

\begin{abstract}
This article aims to investigate how collaborative consumption changes the social context of people in socioeconomic situation of social vulnerability. We carried out a descriptive research, approaching the problem in a qualitative way and using as object ofstudy the case of the Community of Little Prophets (CPP). Data were collected through semi-structured interviews, on the Community's website and in the 2018 Community activity report. Data analysis was performed using the content analysis technique. The results showed that collaborative consumption practices, such as: reuse, shared production of consumer goods, consumption with a focus on sustainability, waste reduction, developed by the participants of the case study have contributed to modify the socioeconomic context of the participants. This was observed in the improvement of the quality of life and nutrition of the participants, income generation process in families, fostering of entrepreneurship and social belonging. Thus, we conclude that collaborative consumption can positively modify the socioeconomic context of people in situations of social vulnerability.
\end{abstract}

Keywords: Collaborative economy. Social change. Sustainable consumption. 


\section{Introdução}

Ao tratarmos de consumo logo nos ocorre a ideia que relaciona a entrega de um bem ou serviço em troca de um dado valor monetário, suprindo a necessidade e expectativas dos agentes da negociação. Segundo McCracken (2003), o consumo moderno é, acima de tudo, um artefato histórico, cujas características atuais são o resultado de vários séculos de profunda mudança social.

Atualmente, há um movimento no sentido do consumo compartilhado e colaborativo. Para Botsman e Rogers (2011), cotidianamente as pessoas estão usando o consumo colaborativo, através do compartilhamento tradicional, escambo, empréstimo, negociação, locação, doação e troca, seja essas ações realizadas por meio da tecnologia ou em uma comunidade. Esse consumo proporciona às pessoas os benefícios do acesso a produtos e serviços sem objetivamente obter a propriedade. Os autores ainda afirmam que o consumo colaborativo oportuniza a criação de novas amizades e também contribui para uma consciência cidadã dos consumidores, aspectos que tornam possível vislumbrar uma perspectiva de crescimento desse modo de consumo.

É bem verdade que as práticas de consumo colaborativo têm sido impulsionadas pela tecnologia e pelo surgimento de redes sociais e aplicativos cada vez mais específicos, que disponibilizam conteúdos diversos e são customizados para o mercado alvo. Nesse sentido, ainda segundo Botsman e Rogers (2011), surgiram nos últimos anos modelos de negócios como o Airbnb e Zipcar que viabilizam o consumo colaborativo em suas plataformas.

Segundo a Confederação Nacional de Dirigentes Lojistas, o número de pessoas dispostas a adotar práticas de consumo colaborativo cotidianamente, nos próximos dois anos, cresceu de 68\% em 2018 para $81 \%$ em 2019. Foram ouvidos mais de 800 brasileiros, nas 27 capitais do país (MEIO \& MENSAGEM, 2019). Dados como estes reforçam um novo padrão de consumo que não é mais caracterizado apenas pela busca de uma satisfação pessoal, de pertencimento, status ou a aquisição de bens e serviços, em sua maioria desnecessários, motivada pelos desejos dos sujeitos (BAUMAN, 2008), o que nos remete ao consumismo.

$\mathrm{Na}$ abordagem de Botsman e Rogers (2011), sobre o consumo colaborativo, o hiperconsumo deixa de ser o norteador das motivações humanas e o esgotamento dos recursos naturais passa a ser um fator de preocupação, uma oportunidade de repensar o relacionamento entre as pessoas e os produtos. Segundo McCracken (2003), as pessoas estão dispostas a constituir novas experiências de consumo em que a acumulação de bens, a 
preocupação com esgotamento de recursos e o legado para gerações futuras são temas em evidência e recorrentes ao consumir produtos e serviços. Esse comportamento imprime um significado mais social para o consumo e coloca o compartilhamento como uma alternativa, um ato comum entre as pessoas que promove níveis de solidariedade, estabelecendo assim, de acordo com Pizzol (2015, p. 18), um "novo modelo, com novos padrões de consumo que está intimamente ligado na sustentabilidade, redução do hiperconsumo e do desperdício e no reuso dos produtos".

Apesar do apresentado, há estudos como os de Druck (2013) e Pochmann (2001) que entendem esse novo modelo de consumo como um modo resultante do modelo capitalista, argumentando que muitos dos negócios que envolvem o consumo colaborativo na verdade visam ao lucro. Autores como Harvey (1993), Pochmann (2001) e Druck (2013) estão conscientes de que há uma ótica individualista do consumo ou um incentivo ao consumo exacerbado proposto por um modelo precarizado e capitalista. Podemos até achar paradoxal consumir de forma compartilhada algo com objetivos individuais, mas os princípios do consumo compartilhado ou colaborativo difundidos, em sua maioria, baseiam-se neste tipo de modelo no qual o uso de produtos particulares de forma compartilhada pode gerar um retorno financeiro. Mas o que se objetiva neste estudo é uma nova perspectiva de consumo, pouco explorada na literatura, em que há um modelo sustentável resultante da inter-relação de atores sociais, pressupondo a existência de uma consciência individual ao considerar o indivíduo como cidadão, como afirma Silva (2012).

Mas será que o consumo colaborativo visa apenas uma recompensa monetária? Há ganho social nas práticas de consumo colaborativo? Ao compartilharmos, visamos o benefício comum ou só a lucratividade? É possível desenvolver um novo contexto social para pessoas em situação de vulnerabilidade socioeconômica através do consumo colaborativo? Com relação ao último questionamento apresentado, observamos que há uma escassez de estudos científicos em relação à abordagem do consumo colaborativo em classes sociais mais baixas ou com pessoas em situação de vulnerabilidade social. Há artigos em que o objeto estudado são classes sociais mais elevadas, como o de Orsi (2017), por exemplo, que se preocupa com a relação da colaboração entre pessoas pertencentes às classes $\mathrm{A} e$ B. Indivíduos que possuem poder de compra e têm renda estabelecidas de maneira formal.

Assim, o artigo em questão visa estudar um outro contexto, o de pessoas em situação socioeconômica de vulnerabilidade social, caracterizado por pessoas que são estigmatizadas, esquecidas e sobrevivem às margens da sociedade sem dispor de poder de compra e renda, 
vivendo em situação de risco social. Bruseke (2007) afirma que a vulnerabilidade social envolve uma conjunção de fatores, sobrepostos de diversas maneiras e em várias dimensões, de modo a tornar o indivíduo ou grupo mais suscetível aos riscos e contingências. Já Rocha (2003) ressalta a importância de se criar mecanismos para identificar as várias dimensões que compõem situações de vulnerabilidade. Mas por que estudar o consumo colaborativo em um ambiente teoricamente desfavorável a ele? Diante de um quadro de instabilidade econômica e redução do poder de compra, ações de compartilhamento podem configurar uma alternativa para as pessoas que estão nesse contexto. Nesse sentido, Singer (2018) afirma que práticas caracterizadas pela igualdade de direito, produção e posse são de suma importância para o desenvolvimento do espírito empreendedor como também no processo de geração de renda, o que pode contribuir para a melhoria das condições sociais de pessoas em situação de vulnerabilidade.

Considerando o contexto de comunidades, Petruskeviciute (2019) afirma que, ao utilizar a inovação social, design ecológico, reutilização de resíduos e aprendizagem ao ar livre para co- desenvolver e entregar soluções locais a longo prazo, as comunidades estabelecem prioridades e necessidades locais; aumentam a inclusão ativa, o equilíbrio entre produção e consumo através do uso de fontes sustentáveis, trabalhando estilos de vida dos cidadãos envolvidos nessas transformações através de práticas criativas de colaboração em redes locais. Podemos observar que alguns dos aspectos abordados por Petruskeviciute (2019) podem ser encontrados nos tipos de consumo colaborativo Mercados de redistribuição e Estilo de vida colaborativo definidos por Botsman e Rogers (2011). Assim, questionamos sobre a aplicação do consumo colaborativo como uma forma de resgate de populações ou comunidades vulneráveis, e se é possível relacionar uma melhoria no contexto socioeconômico dessas pessoas por meio de práticas de consumo colaborativo.

Diante do apresentado, temos como objetivo geral: investigar como o consumo colaborativo modifica o contexto social de pessoas em situação socioeconômica de vulnerabilidade social. Para a consecução do objetivo, realizamos a pesquisa na Comunidade Pequenos Profetas (CPP) cujos participantes são pessoas que se encontram em situação de vulnerabilidade social. Dessa forma, definimos os seguintes objetivos específicos: descrever a comunidade Pequenos Profetas; identificar as ações que envolvem o consumo colaborativo desenvolvidas na Comunidade Pequenos Profetas e as mudanças observadas na vida dos participantes decorrentes dessas ações

Este estudo justifica-se por haver na literatura poucos estudos que abordem o consumo colaborativo no contexto de consumidores em situação socioeconômica de 
vulnerabilidade social. Dessa forma, esta pesquisa pode contribuir para preencher essa lacuna na literatura promovendo um melhor entendimento das práticas de consumo colaborativo no contexto que o estudo propõe evidenciar. Os resultados do trabalho podem apontar uma nova forma de aplicação do consumo colaborativo não usual ou ainda pouco estudada.

\section{Metodologia}

Esta é uma pesquisa exploratória descritiva com abordagem qualitativa. A pesquisa é exploratória porque tem como objetivo proporcionar maior familiaridade com o problema, com vista a torná-lo mais explícito ou a construir hipóteses (GIL, 2010). Também pode ser definida como descritiva tendo em vista que tem como objetivo primordial a descrição de características de determinada população ou fenômeno (GIL, 2010), no caso da comunidade Pequenos Profetas. A pesquisa também tem uma abordagem qualitativa, haja vista que busca uma relação dinâmica entre o mundo objetivo e a subjetividade do sujeito. $O$ ambiente natural é a fonte direta para coleta de dados e o pesquisador é o instrumento-chave. Além disso, não requer o uso de métodos e técnicas estatísticas (SILVA; MENEZES, 2001).

Como estratégia de investigação utilizamos um estudo de caso único. Segundo Mattar (2005, p. 88-89), o estudo de caso é "um método muito produtivo para estimular a compreensão e sugerir hipóteses e questões para a pesquisa. O método do estudo de caso pode envolver exame de registros existentes, observação da ocorrência do fato, entrevistas semiestruturadas, entrevistas não estruturadas, etc... Conforme já citado, o caso que servirá como campo empírico deste estudo será a Comunidade Pequenos Profetas em Recife, pois ele oportuniza o estudo do fenômeno pesquisado já que envolve pessoas que se encontram em situação de vulnerabilidade socioeconômica e nele são desenvolvidas práticas de consumo colaborativo.

Para a coleta de dados, adotamos a entrevista semiestruturada com a utilização de um roteiro com questões relacionadas ao perfil do entrevistado, às ações de consumo colaborativo desenvolvidas na Comunidade e às mudanças decorrentes do uso de práticas de consumo colaborativo na vida dos participantes da Comunidade dos Pequenos Profetas. Nesse sentido, entrevistamos o gestor da Comunidade, criador do projeto, e a assistente social. Também utilizamos na coleta de dados informações disponíveis no site da Comunidade e no relatório de suas atividades do ano de 2018 que contém dados de auditorias e consultorias 
internacionais sobre os impactos do projeto na vida dos participantes. As entrevistas tiveram duração média de 30 minutos.

Os dados foram analisados por meio da técnica de análise de conteúdo que, segundo Bardin (2011, p. 47), é um conjunto de técnicas de análise das comunicações visando a obter, por procedimentos sistemáticos e objetivos de descrição do conteúdo das mensagens, indicadores (quantitativos ou não) que permitam a inferência de conhecimentos relativos às condições de produção/recepção (variáveis inferidas) dessas mensagens.

As entrevistas, o site e o relatório de atividades foram analisados tendo como tema-eixo os objetivos da pesquisa. Inicialmente fizemos a leitura desse material buscando identificar as palavras e frases que ajudassem a responder os objetivos da pesquisa; em seguida selecionamos as partes dos textos que melhor permitiram fazer um contraponto dos achados da pesquisa com a teoria.

\section{Análise dos Resultados}

Nos tópicos a seguir, descrevemos brevemente sobre a Comunidade Pequenos Profetas, as ações de consumo colaborativo desenvolvidas na comunidade e as mudanças observadas na vida dos participantes decorrentes dessas ações.

\subsection{A comunidade Pequenos Profetas}

O projeto da Comunidade Pequenos Profetas iniciou-se há 30 anos a partir de um sonho de fazer algo e ajudar as pessoas que vivem em contexto de vulnerabilidade social. Hoje o projeto não é apenas do gestor, profissional com formação em nutrição. É uma organização não governamental que atendeanualmente mais de 1000 crianças e adolescentes em situação de vulnerabilidade social. O projeto localiza-se na cidade do Recife/PE - Brasil, e segundo seu relatório de atividades de 2018, conta com um grupo de profissionais que compartilham conhecimentos desenvolvendo diversas atividades multidisciplinares que envolvem atividades educativas, artísticas, culturais, esportivas, ambientais e gastronômicas, além do acompanhamento contínuo através de assistência jurídica, psicológica, social, nutricional e de promoção à saúde, a fim de promover a cidadania em um público em risco social. Nesse sentido, fica evidenciado que o estilo de vida colaborativo, definido por Botsman e Rogers (2011), que consiste na interação de pessoas com interesses similares e com o desejo de compartilhar ativos menos tangíveis, como: conhecimento, recursos, espaço, habilidades e 
tempo, intensificando assim a interação social e a entrega de valor para esses indivíduos, é fator norteador das ações realizadas na Comunidade.

O gestor do projeto, na entrevista, definiu pessoas em vulnerabilidade socioeconômica como aquelas que estão em situação de risco social. Para ele, são as pessoas que advém das comunidades empobrecidas com baixo IDH e que são invisíveis para o poder público e para sociedade. São elas: usuários de drogas, prostitutas, mulheres com histórico de violência doméstica ou que sofreram violência sexual, pessoas em estado de desnutrição, famílias de baixa renda ou que não tenham renda nem poder de compra. Para Rocha (2003), mais do que dizer o que é vulnerabilidade socioeconômica, requer-se definir instrumentos e referências para buscar as verdades da realidade perceptível e das que não aparecem nas estatísticas, pois há uma multidimensionalidade da vulnerabilidade.

A CPP tem o reconhecimento de várias entidades internacionais, entre elas a Organização das Nações Unidas, por seu trabalho voltado aos direitos humanos, além de parcerias com institutos internacionais que financiam o projeto. Considerando suas ações no público evidenciado, o projeto é ganhador de vários prêmios de gestão e promoção da igualdade de direito e social de seus participantes. O mais recente, segundo o site a Comunidade, foi o prêmio Direitos Humanos Nacho de La Mata 2019 - Abogacía Española, recebido no mês de dezembro de 2019 na Espanha.

Alguns dos dados que exemplificam e ilustram o resultado alcançado pelo projeto podem ser encontrados no relatório anual da CPP 2018, a seguir apresentados:

a) 1000 atendimentos psicossociais, com 160 visitas domiciliares e 300 famílias atendidas em 12 reuniões com pais e responsáveis;

b) 350 acompanhamentos nutricionais de crianças e adolescentes;

c) 100 atendimentos relacionados a primeiros atendimentos de enfermagem;

d) 100 atendimentos odont ológicos em duas ações;

e) 152 oficinas de artes com 1594 participações;

f) 182 rodas de leituras sobre Direitos Humanos.

Segundo o gestor, para que os participantes possam usufruir das ações desenvolvidas na CPP, eles devem passar por uma triagem de acordo com o estatuto da organização. Conforme informação disponível no site da Comunidade Pequenos Profetas, a entidade trabalha com o propósito de atenuar, combater e reverter os problemas enfrentados pelo público evidenciado. É nesse sentido que a pesquisa busca explorar o contexto das ações 
do projeto a fim de investigar como o consumo colaborativo modifica o contexto social de pessoas em situação socioeconômica de vulnerabilidade social.

3.2 Ações que envolvem o consumo colaborativo desenvolvidas na Comunidade Pequenos Profetas e as mudanças observadas na vida dos participantes decorrentes dessas ações

Como dito anteriormente, ações multidisciplinares são oferecidas cotidianamente, mas com a finalidade de realizar o objetivo desta pesquisa, focamos a atenção naquelas com mais aderência ao conceito de consumo colaborativo e que diante dos dados contribuem para modificar o contexto socioeconômico de vulnerabilidade social de seus participantes. Entre todas as atividades, apresentamos quatro ações referentes à prática de consumo colaborativo desenvolvidas na CPP: Hortas verticais, Telhado Eco produtivo, Gastronomia Social e Faça e venda.

O projeto Hortas Verticais foi criado em abril de 2010 com o objetivo de minimizar a extrema pobreza em que se encontravam os jovens atendidos pela Comunidade Pequenos Profetas e suas famílias. O projeto abrange toda a comunidade ribeirinha que mora em palafitas e se destina ao aproveitamento das garrafas pet que são descartadas irregularmente no rio Capibaribe. Os participantes do projeto recolhem as garrafas, elas são higienizadas, cortadas ao meio e adaptadas para a produção de horticultura, onde são cultivadas 18 tipos de hortaliças. Dessa forma, eles trabalham aspectos defendidos por Pizzol (2015) como o reuso de produtos.

Segundo o gestor, esses participantes já retiraram do rio mais de 1 tonelada de garrafas pet descartadas de forma irregular. É a consciência ambiental sendo valorizada e impulsionada nesse público em vulnerabilidade com baixo índice de desenvolvimento humano. Eles contribuem para uma sociedade que valoriza o meio ambiente e preocupa-se com o esgotamento dos recursos naturais, utilizando cotidianamente práticas de consumo colaborativo, como: técnicas de produção compartilhada de alimentos, produção e reuso de materiais, a fim de promover o consumo desses alimentos em igualdade de direitos (BOTSMAN; ROGERS, 2011). Tais alimentos possibilitaram a inclusão na dieta dos participantes de alimentos que eles nunca tiveram contato ou tão pouco a oportunidade de consumir.

Segundo o relatório de atividades de 2018, os dados apontam no sentido de que a horta vertical contribui substancialmente nos índices de qualidade de vida das famílias, sendo primordial do ponto de vista nutricional como também na melhoria do hábito de 
consumo das pessoas, na economia das famílias e até na manutenção e/ou melhoria da saúde e prevenção de doenças. Durante o ano, foram realizadas 167 ações, com 1158 participações. As famílias participantes das ações receberam cerca de 500 doações de hortaliças, frutas e verduras, além do consumo no projeto.

Outro projeto de grande destaque no que se refere aos impactos produzidos é o Telhado Eco Produtivo. É um projeto inovador pensado em 2015 após uma série de arrombamentos na sede do projeto Comunidade Pequenos Profetas que resultaram em roubos de equipamentos e prejuízo financeiro de mais de 47 mil reais. Com patrocínio da PETROBRAS, através do Programa Petrobras Socioambiental, além do suporte financeiro e incentivos das instituições: Förderverein Straßenkin do Recife, Aktions Kreis Pater Bedader e V.-Havixbeck da Alemanha, Solvida Rede de Parceiros no Brasil, BMZ e do Ministério de cooperação alemã, foi possível viabilizar esse projeto. De acordo com o relatório de atividades e informações do site da CPP, são mais de 1.500 pessoas beneficiadas diretamente em localidades com baixo IDH no Recife, com uma produção anual estimada em mais de meia tonelada de alimentos.

A obra do Telhado Eco Produtivo foi iniciada em maio de 2016 e conta com tecnologia japonesa, sistema de irrigação, energia solar e piso em madeira certificada. É um projeto que, além de trabalhar aspectos como os já observados na Horta vertical, promove o desenvolvimento comunitário, a democratização do acesso à produção orgânica, à alimentação saudável e à gastronomia social, ações em consonância com o estilo de vida colaborativo observado por Botsman e Rogers (2011). São cerca de 400 metros quadrados de hortas orgânicas, montadas em estruturas ecológicas de madeira, em cima de um casarão antigo, no coração do Recife, onde funciona a sede da Comunidade Pequenos Profetas (CPP). O telhado é muito mais que um espaço físico; é um espaço para se discutir e fomentar a inclusão, democratização do acesso a conceitos de produção orgânica, sustentável e alimentação saudável, é o que afirma os idealizadores e o que comprovam os dados analisados.

No projeto ocorrem ações de fomento à geração de renda através de feirinhas a preço populares, realizadas quinzenalmente com os produtos cultivados de forma orgânica, produzidos pelos próprios moradores a partir de suas experiências no projeto da CPP. Os participantes recebem apoio técnico para produzir suas próprias hortas em casa, e contam com a cozinha apoio para aulas de gastronomia orgânica e alimentação saudável, 
o que promove a participação coletiva na produção e a aquisição de conhecimento para desenvolver um meio para gerar renda.

Pensando no uso dos materiais produzidos ou do que geralmente iria para o lixo como resíduos de frutas e verduras, surgiu o projeto da Gastronomia social. O projeto utiliza o alimento como ferramenta de transformação social, através da melhoria dos cardápios e inclusão de novos hábitos alimentares e outros tipos de alimentos produzidos pelos participantes. De acordo com o relatório de 2018, foram realizadas 104 oficinas, com 246 participações. Isso é replicado seja nas casas dos participantes, com o conhecimento das hortas verticais, seja no projeto do Telhado Eco Produtivo conscientizando as pessoas e levando um impacto positivo para a vida dos participantes, pois, ao alimentar-se de forma adequada, eles resgatam aspectos como autoestima, motivação e depressão. Segundo o gestor, isso modifica a forma como eles se veem no ambiente social.

O relatório confirma ainda que a inclusão de novos hábitos de consumo no projeto acarretou a melhoria dos índices nutricionais dos participantes bem como na geração de renda, pois dispõem de aulas de gastronomia e desenvolvem essas práticas também nas suas casas e levam como experiência profissional para suas vidas. Como afirma o gestor ao dizer: "Quando vejo um adolescente daqui, na minha área que é a gastronomia, que eu treinei, está em um bom restaurante trabalhando, carteira assinada e com seu dinheiro, procurando estudar, fazendo cursos, abrindo seus horizontes, quem não tinha nenhuma perspectiva na vida, isso nos motiva".

Na busca pela diferenciação de produtos alimentícios conhecidos no mercado como coxinhas e empadas, por exemplo, foi criado o programa Faça e venda e a Oficina de ovos de Páscoa. Nesses projetos, eles customizam velhas receitas e produzem de forma diferenciada diversos alimentos, utilizando a cozinha compartilhada, além dos materiais anteriormente produzidos em algum projeto dentro da CPP. O uso sustentável de materiais como cascas de verduras na produção compartilhada de bens de consumo é transformador do ponto de vista nutricional e da colaboração. Além disso, esses programas contribuem para gerar renda. Tal fato promove melhorias e fomenta o comércio na comunidade, pois grande parte dos participantes não têm renda ou recebem menos de um salário mínimo por grupo familiar, foi o que explicou a assistente social. Essas ações resgatam o valor social, proporcionam dignidade dentro do processo de pertencimento e no convívio social. 


\section{Conclusão}

O caso aqui estudado, a Comunidade Pequenos Profetas (CPP), possibilitou fazer um contraponto entre os projetos desenvolvidos na CPP e o conceito de consumo colaborativo, tentando preencher uma lacuna identificada na literatura que diz respeito à relação entre consumo colaborativo e melhorias efetivas na vida de pessoas em contexto de vulnerabilidade socioeconômica.

Os resultados evidenciam as seguintes conclusões:

1. Os projetos desenvolvidos na Comunidade CPP envolvem práticas de consumo colaborativo relacionadas aos tipos Mercados de redistribuição e Estilo de vida colaborativo. Nesses projetos, os participantes da comunidade reutilizam produtos, compartilham espaços e colaboram uns com os outros na produção de produtos para consumo próprio, como hortaliças e outros alimentos, ou ainda para a geração de renda.

2. As ações realizadas na CPP que promovem o reuso de materiais amplamente difundido na comunidade, interação social no que se refere à colaboração e à produção compartilhada de bens de consumo, geram a consciência ambiental e promovem a sustentabilidade e a cidadania, são um forte elemento construtivo na promoção de melhorias efetivas na comunidade.

3. Nesse sentido, os dados disponibilizados no relatório de atividades de 2018 e as entrevistas realizadas indicam melhorias na questão do pertencimento, no sentido de coletividade e cooperação, na felicidade em contribuir com sua comunidade e com os entornos, na alimentação, na capacidade de produzir algo que possa ser comercializado, tudo isso contribuindo para melhorar a qualidade de vida dos participantes da Comunidade.

4. Assim, o consumo colaborativo pode modificar de forma positiva o contexto socioeconômico de pessoas em situação de vulnerabilidade social. Tal fato abre uma discussão acerca do tema e sobre o olhar dos pesquisadores em torno das práticas de consumo colaborativo no contexto de pessoas empobrecidas e em risco social. 


\section{Referências}

BARDIN, L. Análise de conteúdo. São Paulo: Edições 70, 2011.

BAUMAN, Z. Vida para consumo: a transformação das pessoas em mercadorias. Rio de Janeiro: Jorge Zahar, 2008.

BOTSMAN, R.; ROGERS, R. O que é meu é seu cada vez mais: a ascensão da economia colaborativa. 1. ed. São Paulo: Bookman, 2011.

BRUSEKE, F. J. Risco e contingência. Revista Brasileira de Ciências Sociais, São Paulo, v. 22, n. 63, 2007. Disponível em: http://www.scielo.br/pdf/rbcsoc/v22n63/a06v2263.pdf.

DRUCK, G. A precarização social do trabalho no Brasil:alguns indicadores. In:ANTUNES, R (org.). Riqueza e miséria do trabalho no Brasil II. São Paulo: Boitempo, 2013. cap. 4, p. 55-73. Disponível em: https://edisciplinas.usp. br/pluginfile.php/2649414/mod_resource/content/1/Druck\%202013\%20Precariza\%C3\%A7\%C3\%A30\%20 no\%20Brasil.pdf.

GIL, A. C. Como elaborar projetos de pesquisa. 5. ed. São Paulo: Atlas. 2010.

HARVEY, David. A condição pós-moderna: uma pesquisa sobre as origens da mudança cultural. Tradução de Adail Ubirajara Sobral e Maria Stela Gonçalves. 3. ed. São Paulo: Edições Loyola, 1993.

MATTAR, F. N. Pesquisa de marketing: metodologia, planejamento. v. 1, 6. ed. São Paulo: Atlas, 2005.

MCCRACKEN, G. Cultura e consumo: novas abordagens ao caráter simbólico dos bens e das atividades de consumo. Rio de Janeiro: Ed. Mauad, 2003.

MEIO \& MENSAGEM. Economia colaborativa cresce, mas confiança ainda é barreira. Disponível em: https:// www.meioemensagem.com.br/home/midia/2019/08/15/economia-colaborativa-cresce-mas-confiancaainda-e-barreira.html. Acesso em: 15 out. 2019.

ORSI, D. F. O. O consumo colaborativo em estratos econômicos A e B na cidade de São Paulo - SP. 2017. 139 f. Dissertação (Mestrado em Administração) - Universidade Metodista de São Paulo, São Paulo, 2017. Disponível em: http://tede.metodista.br/jspui/bitstream/tede/1707/2/Daniella\%20Fernandes\%20de\%20 Oliveira\%200rsi.pdf.

PETRUSKEVICIUTE, L. From the city of gaps to the city of wellness: the case study of Dot to Dot community garden in Maryhill, Glasgow. Visions for Sustainability, v. 11, p. 37-46, 2019. Disponível em: https://www. semanticscholar.org/paper/From-the-city-of-gaps-to-the-city-of-wellness\%3A-The-Petruskeviciute/ fabe5cd329ae01aa07591652dcf58829d04d4065.

POCHMANN, M. O emprego na globalização: a nova divisão internacional do trabalho e os caminhos que o Brasil escolheu. São Paulo: Bomtempo, 2001.

PIZZOL, H. O. D. Proposição de uma escala para mensuração do consumo colaborativo: compreendendo o compartilhamento de bens e a sua relação com os valores pessoais. 2015. 166 f. Dissertação (Mestrado em Administração e Negócios) - Faculdade de Administração, Contabilidade e Economia, Pontifícia Universidade Católica do Rio Grande Do Sul, Porto Alegre, 2015. Disponível em: https://repositorio.pucrs.br/dspace/ bitstream/10923/7350/1/000469987-Texto\%2bCompleto-0.pdf.

ROCHA, S. Pobreza no Brasil: afinal, do que se trata?. Rio de Janeiro: FGV, 2003, 244 p. 
SILVA, M. Consumo sustentável: a articulação de um constructo sob a perspectiva do desenvolvimento sustentável. RECADM, v. 11, n. 2, jul./dez. 2012. Disponível em: http://www.periodicosibepes.org.br/index. php/recadm/article/view/1052/675.

SILVA, E. L.; MENEZES, E. M. Metodologia da pesquisa e elaboração de dissertação. 3. ed. Rev. Atual. Florianópolis: Laboratório de Ensino a Distância da UFSC, 2001.

SINGER, P. Ensaios sobre economia solidária. Coimbra: Ed. Almedina S.A., 2018. 